\begin{tabular}{|c|c|}
\hline \multirow{3}{*}{ 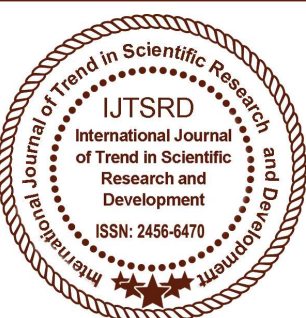 } & $\begin{array}{l}\text { International Journal of Trend in Scientific } \\
\text { Research and Development (IJTSRD) }\end{array}$ \\
\hline & Access Journal \\
\hline & ISSN No: 2456 - 6470 | www.ijtsrd.com | Volume - 2 | Issue -3 \\
\hline
\end{tabular}

\title{
Abnormal Driving Behaviors detection with smart phones
}

\author{
${ }^{1}$ Dr. B. Srinivasa Rao, ${ }^{2}$ U. Sri Devi, ${ }^{3}$ K. Sri Satya Harsha, ${ }^{4}$ A. Rakesh, ${ }^{5}$ K. Muralidhar \\ ${ }^{1}$ Professor \& Head of Department, \\ $1,2,3,4,5$ Department of Computer Science,
}

Dhanekula Institute of Engineering and Technology, Gangur, Andhra Pradesh, India

\begin{abstract}
Abnormal driving behaviors detection is a key stone for reducing accidents. There are several existing works for abnormal driving detection which uses high cost sensors to check whether the driver is in normal state or in drunken state. In this paper we would like to introduce a low cost technique for detecting abnormal driving behaviors with the use of some in built Smartphone sensors. With the information collected from the sensors we will employ the machine learning technique (SVM and KNN) to extract the features and for generating a classifier model. We will send the alerts if abnormality is detected.
\end{abstract}

Keywords: Abnormal Driving behaviors; SVM (Support Vector Machine); KNN(k-Nearest Neighbors); Smart phone sensors; sending alerts

\section{INTRODUCTION}

According to Annual Global Road Crash Statistics 2017, nearly 1.3 million people die in accidents each year which indicates that on average 3,287 deaths a day. In addition to that 20-50 million are injured are disabled. It also states that road accidents is the $9^{\text {th }}$ leading cause for deaths. If corresponding actions are not taken road accidents are predicted to be the $5^{\text {th }}$ leading cause for deaths.

Although there has been many works on abnormal driving behaviors they focus mainly on detecting status of driver i.e. drunk, drowsy or fatigue. Since smart phones are used widely now-a-days, we would like to develop an android application which detect the abnormal driving behavior and sends an alert if any such case is found.

Going along this direction we considered the Smartphone sensors (accelerometer and orientation sensors) which are in-built sensors for many android phones. With the help of these sensors we extract the features for all abnormal driving behaviors which will be useful for distinguishing the abnormal driving behaviors from normal ones.

This has a wide scope for development if these results are monitored by Government for identifying rash driving actions which leads to accidents. The government can also collect the fines from the people who are identified to be driving abnormal. It can also be used by the car owners for analyzing the status of their drivers. According to [1] there are six types of abnormal driving behaviors which are Weaving, Swerving, Side-slipping, Fast U-turn, turning with wide radius, Sudden braking. These six types of driving behaviors have unique patterns. In this work, firstly we will collect the data from some experienced drivers by employing all the types of abnormal driving behaviors through Smartphone. We perform machine learning to generate a classifier model which helps in extracting the features for detecting abnormal driving behaviors.

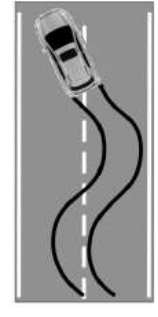

(a)

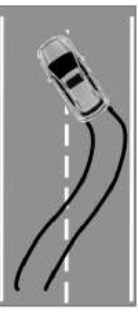

(b)

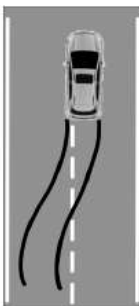

(c)

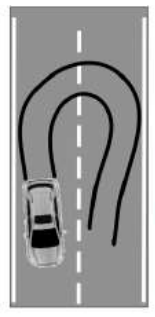

(d)

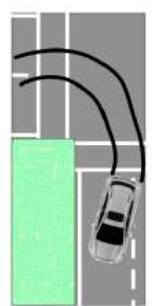

(e)

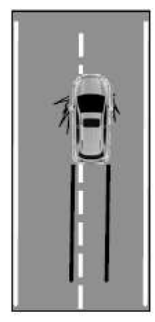

(f)

Fig 1 :(a) weaving, (b)swerving, (c)Side slipping, (d)

Fast U-turn, (e) Turning with a wide radius, (f) Sudden braking 


\section{RELATED WORK}

Nidhi Kalra et al. [2014] have studied that in today's life, everyone seems to be in hurry to achieve their destination as quickly as doable. Therefore individuals advisedly or accidentally take harsh driving events like unforeseen acceleration, unforeseen turns or unforeseen brakes that more lead them to accidents or maybe loss of their lives. And it's a typical belief that if person behaviour was being monitored, it might be comparatively safer. To watch driver behaviour numerous sensors were getting used either deployed within the automotive, wayside or inherent in Smartphone.This paper conjointly provides some analysis directions that numerous researchers will explore.

Prarna Dhar et al. [2014] have studied the Unsafe driving primarily includes driving either headlong or driving below the Influence (DUI) of alcohol, may be a major reason for traffic accidents throughout the planet. During this paper, we advise an extremely system that helps at early detection and alert of dangerous vehicle manoeuvres usually associated with rash driving. The complete system needs solely an itinerant which can be placed in vehicle-and with its inherent measuring system and orientation sensing element. When putting in a program on the itinerant, it'll cypher accelerations supported sensing element readings and compares them with typical unsafe driving patterns extracted from real driving tests.

Rachana Daigavane et al. [2015] has studied and discovers the most causes of accidents and so provides risk assessments thus, serving to cut back careless driving and enforce safe driving practices. Showing determination behaviour and energetic pursuit of your ends was presently a causal agency of traffic in an urban centre. Awareness and encourage driver safety square measure the measures that square measure further, we have a tendency to square measure shall propose a decent arrangement that uses detection system and management of the vehicle. For the foremost half, drivers aren't aware that they offer disposition to behave sharply activity found within the normal course of events. Among the factors concerned in driving, particularly the motive force, the vehicle, and also the surroundings, the human issue is that the most relevant and most troublesome to characterize. This project wasn't solely helpful for the motive force's behaviour detection however conjointly offer reconstruction and investigation of accidents and during this thanks to cut back the risks and dangers for the driver.

\section{SYSTEM DESIGN}

In this section the design of our proposed system which detects abnormal driving behaviors using smart phones is described. Our system does not require any additional hardware like alcohol sensors or video capturing devices for detecting abnormal driving behaviors.

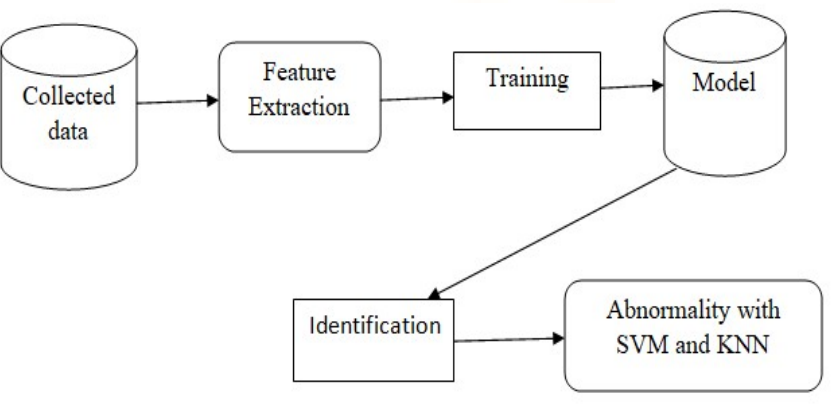

Fig 2: System architecture

Figure 2 shows the architecture of our system. We divide the architecture of our system into two phases which are offline phase which includes modeling abnormal driving behaviors and online phase which includes monitoring of abnormal driving behaviors.

In the offline part we train a classifier model using machine learning technique basing on the data collected. In the feature extraction different features are extracted from the collected data using accelerometer and orientation sensor. After the feature extraction the features are trained in the training part and generate a classifier model. The final output of the offline phase is the model which is stored in a database.

In the online phase to monitor the abnormal driving behaviors, the developed application is installed in the smart phones which sense the values from accelerometer and orientation sensors. After sensing the values from the real time driving, co-ordinates are arranged along the direction of the vehicle from the beginning to ending of driving phase. Afterwards in the identification phase we extract the features from real time driving and employ the classifier model to check whether abnormal driving behaviors occurred. If any of the abnormal driving behaviors are detected then an alert message will be sent to the specified number. For the features extraction we used support vector machine (SVM) and k-nearest neighbors $(\mathrm{KNN})$. 


\section{MODULES}

\section{Data collection from Smartphone sensors}

We collect the data from Smartphone sensors through an android application which uses accelerometer and orientation sensor. We align the co-ordinate axes in the direction of vehicle through which we can monitor the driving behaviors by retrieving the values from sensors.

\section{Low Pass Filtering}

The collected data may have some noise. To remove noise from the collected data there are many noise removal techniques. In this paper we performed low pass filtering to remove high frequency noise.

\section{Analyzing Patterns}

After filtering the data we analyze the patterns of abnormal driving behaviors by considering the factors like acceleration, range, mean and standard deviation. The unique patterns through which we can analyze different abnormal behaviors are as follows:

\section{i. Weaving:}

In this pattern the acceleration along $\mathrm{X}$-axis $\left(\operatorname{acc}_{\mathrm{x}}\right)$ fluctuates drastically for a period of time where as acceleration along $\mathrm{Y}$-axis $\left(\mathrm{acc}_{\mathrm{y}}\right)$ remains constant. This implies standard deviation and range of $\operatorname{acc}_{\mathrm{x}}$ are high.

\section{ii. Swerving:}

In this pattern the acceleration and orientation along $\mathrm{X}$-axis varies for a short period of time which implies that the range of $\operatorname{acc}_{\mathrm{x}}$ and ori $\mathrm{x}_{\mathrm{x}}$ are high where $\mathrm{acc}_{\mathrm{y}}$ and ori remains soft.

\section{iii. Side slipping:}

In this pattern $\mathrm{acc}_{\mathrm{y}}$ falls down sharply which indicates that range of $\mathrm{acc}_{\mathrm{y}}$ is large. Moreover the value of $\mathrm{acc}_{\mathrm{x}}$ will not be zero and will be based on the side that vehicle slips i.e. either right or left.

\section{iv. Fast U-turn:}

In this pattern the value of $\operatorname{acc}_{\mathrm{x}}$ will increase at the beginning of U-turn and remains constant for a period of time and then decreases. This implies that the range of $\operatorname{acc}_{\mathrm{x}}$ is large and same is the case with ori $\mathrm{x}_{\mathrm{x}}$.

\section{v. Turning with a wide radius:}

In this pattern $\operatorname{acc}_{\mathrm{x}}$ and ori $\mathrm{x}_{\mathrm{x}}$ changes for a long period of time where as the values of ori $\mathrm{y}_{\mathrm{y}}$ and $\mathrm{acc}_{\mathrm{y}}$ are nearly equal to zero but not zero. This implies that range of $\operatorname{acc}_{\mathrm{x}}$ and ori $\mathrm{x}_{\mathrm{x}}$ is large.

\section{vi. Sudden braking:}

In this pattern $\operatorname{acc}_{\mathrm{x}}$ and ori $\mathrm{x}_{\mathrm{x}}$ remains constant where as the value of $\operatorname{acc}_{\mathrm{y}}$ decreases drastically. The value of $\operatorname{acc}_{\mathrm{y}}$ varies from beginning to ending. This implies that range of $\mathrm{acc}_{\mathrm{y}}$ is large.

\section{Detecting abnormal driving behaviors with smart phone:}

The figure 5 shows user interface of our application. We have two phases in our application namely analysis and automatic.
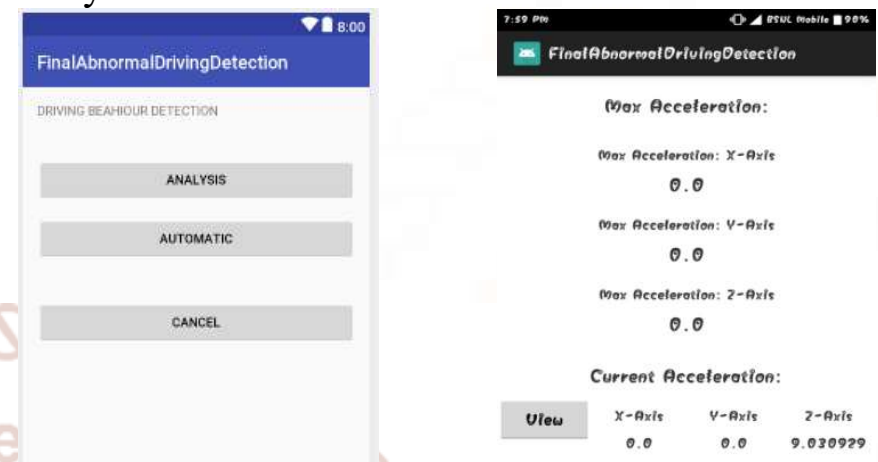

\section{$\triangleleft \quad \circ \quad \square$}

(a)

(b)

Fig: (a) overview of application (b) screen showing acceleration.

The result of analysis phase will give the count of abnormal driving behaviors observed during the driving from beginning to ending. The automatic phase will send an alert automatically to the specified number if any type of abnormal driving behaviors is detected.

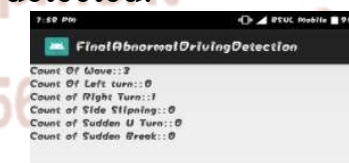
(c) Result of analysis phase (d) Result of automatic phase.

\section{CONCLUSION}

In this paper we developed an application to detect abnormal driving behaviors with the help of smart phones which does not require any pre deployed infrastructure like alcohol sensor etc. This project has a wide scope for development by including some extra features like:

- $\quad$ Training Set Size

- Traffic Condition 
- $\quad$ Road Type

- $\quad$ Smartphone Placement

- Smartphone Sensors' Sampling Rate

\section{REFERENCES:}

1. U.S.NHTSA. The visual detection of dwi motorists.[Online].Available:http://www.shippd.or g/Alcohol/dwibooklet.pdf

2. World.Health.Organisation. The top ten causes of death.[Online].Available:http://www.who.int/medi acentre/factsheets/fs310/en/

3. M. V. Yeo, X. Li, K. Shen, and E. P. WilderSmith, "Can svm beused for automatic eeg detection of drowsiness during car driving?'Elsevier Safety Science, vol. 47, pp. 115124, 2009.

4. S. Al-Sultan, A. H.Al-Bayatti, and H. Zedan, "Context-aware driver behavior detection system in sintelligent transportaion system," IEEE Trans. on Vehicular Technology, vol. 62, pp. 4264-4275, 2013.

5. J. Paefgen, F. Kehr, Y. Zhai, and F. Michahelles, "Driving behaviour analysis with smartphones: insights from a controlled field study."

6. Y. Wang, J. Yang, H. Liu, Y. Chen, M. Gruteser, and R. P. Martin, "Sensing vehicle dynamics for determining driver phone use," inProc. ACM MobiSys, 2013.

7. H. Han, J. Yu, H. Zhu, Y. Chen, J. Yang, Y. Zhu, G. Xue, and M. Li, "Senspeed: Sensing driving conditions to estimate vehicle speed in urban environments," in Proc. IEEE INFOCOM, 2014.

8. S. Reddy, M. Mun, J. Burke, D. Estrin, M. Hansen, and M. Srivastava, "Using mobile phones to determine transportation modes," ACM Trans. on Sensor Networks, vol. 6, no. 13, 2010.

9. J. Dai, J. Teng, X. Bai, and Z. Shen, "Mobile phone based drunk driving detection," in Proc.PervasiveHealth, 2010, pp. 1-8.

10. M. Fazeen, B. Gozick, R. Dantu, M. Bhukuiya, and M. C.Gonzalez, "Safe driving using mobile phones," IEEE Trans. on Intelligent Transportation Systems, vol. 13, pp. 1462-1468, 2012 